Original article

\title{
RADIOLOGICAL EVALUATION OF CAUDAL VENA CAVA IN DOMESTIC SHORTHAIR CATS WITH REGARD TO RIGHT HEART FAILURE DIAGNOSIS
}

\author{
D. VOSUGH ${ }^{1} \&$ M. N. NAZEM ${ }^{2}$ \\ ${ }^{1}$ Department of Clinical Sciences, ${ }^{2}$ Department of Basic Sciences, \\ School of Veterinary Medicine, Shahid Bahonar University of Kerman, Iran
}

\begin{abstract}
Summary
Vosugh, D. \& M. N. Nazem, 2019. Radiological evaluation of caudal vena cava in domestic shorthair cats with regard to right heart failure diagnosis. Bulg. J. Vet. Med., 22, No 2, 220 226.

Radiology, an imaging technique, is used in checking small animals for cardiovascular and respiratory disorders. Cardiovascular disease such as congestive heart failure, pericardial heart disease, heart worms and disease that cause injury and lesion in the right atrium may lead to an enlarged right side of the heart and as a result cause the enlargement of caudal vena cava (CVC). It is not possible to make a complete comparison of CVC size, due to variety in size of the cats but the ratio of CVC size to the other anatomical structures makes this possibility that we have a better estimation of CVC size. So the aim of this study was to evaluate the ratio of CVC size to aorta (Ao), width of fourth rib (R4) and also the thoracic vertebral length (VL) in 20 male healthy and 20 Domestic Shorthair (DSH) cats with right heart failure (RHF). To this end, the ratio of CVC size to posterior aorta (Ao), the ratio of $\mathrm{CVC}$ size to width of the forth rib, the ratio of CVC size to the length of thoracic vertebrae above the site of trachea bifurcation, CVC/VL of 20 RHF cats to CVC/VL of 20 healthy SHD cats, and also $\mathrm{Ao} / \mathrm{VL}$ ratios were calculated. Statistical analysis showed significant difference in the CVC/Ao and $\mathrm{CVC} / \mathrm{R} 4$ between healthy and RHF cats. CVC/VL was increased in RHF cats in comparison to healthy ones $(\mathrm{P}<0.05)$ while Ao/VL in right heart failure DSH cats was lower than that in healthy DSH cats. The results showed that right heart failure disease in cats may lead to increase in the $\mathrm{CVC} / \mathrm{Ao}, \mathrm{CVC} / \mathrm{R} 4$ and $\mathrm{CVC} / \mathrm{VL}$ parameters in comparison with healthy cats. According to this study, the method that is used to diagnose the right heart failure in dogs could be used for cats too.
\end{abstract}

Key words: cat, caudal vena cava, right heart failure

\section{INTRODUCTION}

The caudal vena cava (CVC) begins on the roof of the abdomen at the level of the last lumbar vertebrae. It passes cranially along the roof of the abdomen to the right of the aorta. CVC enters the thorax by passing through the diaphragm at the caval foramen on the right side of the caudal mediastinum and ends by opening 
into the right atrium (Konig \& Liebich, 2004). CVC is visible on the lateral thoracic radiograph as it runs from the abdominal cavity to the right atrium (Lehmkuhl et al., 1997).

The vena cava is a highly compliant low-pressure capacity vessel (Cina et al., 2006; Sheth \& Fishman, 2007). Its dimensions and dynamics vary with changes in total body water and circulatory blood volume (Lyon et al., 2005), and may be influenced by the systemic and hepatic circulatory status (Braun \& Linggi, 1999; Yeh et al., 2004), the compliance of the hepatic parenchyma (Wachsberg et al., 1998), and changes in the thoracic and abdominal pressure (Natori et al., 1979).

In human medicine, the inferior vena cava (IVC), both the diameter and collapsibility index of the IVC are important for diagnosis of circulation dysfunction. Measurement of the IVC has been reported during diagnosis of patients with cardiac disease classically associated with right-sided congestive heart failure, including severe or chronic congestive heart failure (Braun \& Linggi, 1999; Hollerbach et al., 2001; Cuevas et al., 2006; Kieffer et al., 2006) and pericardial effusion (Curvo-Semedo et al., 2005). Furthermore, the dilatation of the IVC is also observed in patients with cirrhotic portal hypertension (Wachsberg et al., 1998; Cina et al., 2006).

In small animal clinics, dilatation of the CVC is often listed as an indicator of right-sided congestive heart failure (Lehmkuhl et al., 1997; Gidlewski \& Petrie, 2005) and is applied to the diagnosis of dogs with that type of condition, including heartworm disease (Annette et al., 2005), pericardial disease (Gidlewski \& Petrie, 2005), pulmonic stenosis (Jilintal et al., 2006), tricuspid valve regurgita- tion, and dilated cardiomyopathy (Lehmkuhl et al., 1997).

The ratio of $\mathrm{CVC}$ to other anatomic structures may be useful. Therefore, quantitative evaluation has performed to compare the average diameter of the CVC with the diameter of the aorta, length of the $4^{\text {th }}$ thoracic vertebra (Lehmkuhl et al., 1997; Jilintal et al., 2006) and diameter of forth rib (Lehmkuhl et al., 1997) in dogs.

In this study we evaluated a method that has been used by previous studies in healthy and right heart failure dogs (Lehmkuhl et al., 1997; Gidlewski \& Petrie, 2005) in order to find out diagnostic markers to diagnose or evaluate the right heart failure in cats. So, the purpose of this study was to evaluate the CVC size in the male Domestic Shorthair (DSH) cats without overt heart disease in comparison with that in male DSH cats with right heart disease accompanied by physical examination evidence of right heart pressure abnormalities. The ratio of the CVC to aorta (Ao), the ratio of the CVC to the length of the thoracic vertebra above the tracheal bifurcation, and the ratio of CVC to width of the right fourth rib (R4) were estimated.

\section{MATERIALS AND METHODS}

All procedures involving the experimental use of animals were approved by the Animal Ethics Committee, a branch of the Research Council of the Veterinary School in Shahid Bahonar University, Kerman Province, Iran, and administered by the National Animal Ethics Advisory Committee.

This study was done on the 20 healthy male DSH cats and 20 male DSH cats with cardiac diseases commonly associated with right-sided heart failure. The right heart failure cats were diagnosed 
Radiological evaluation of caudal vena cava in domestic shorthair cats with regard to right heart...

after three steps respectively: abnormal heart sounds, electrocardiogram (ECG) and echocardiography. Radiographs showed changes in the overall shape and size of the heart too.

The average age and weight of cats was $5 \pm 1.8$ years and $2.5 \pm 0.5 \mathrm{~kg}$ respectively. They were referred to Shahid Bahonar University Veterinary Medical Teaching Hospital between 2014 and 2015. All male DSH cats were allowed a 2-hour adaptation period in radiograph room before starting the experiment. This protocol was performed at the Veterinary Medicine Faculty, Shahid Bahonar University of Kerman, Iran. In this study there was not used any sedation drugs because of their probably effects on the heart function.

In order to prepare the radiographs, each cat was positioned on the left side on the radiographic cassette. Fore and hindlimbs were extended parallel to the vertebral column towards the tail and head respectively. Sternum was positioned at the same level as the vertebral column. After each cat was placed on radiographic cassette in the middle of the frame, radiographic examinations were performed using $60 \mathrm{kVp}$ and $2.5 \mathrm{mAs}$ with FFD of $80 \mathrm{~cm}$ (Toshiba Xvision EX, Japan). The point of X-ray was focused on the fifth intercostal space.

The following measurements were made from left lateral, inspiratory-phase, thoracic radiographs in each male DSH cat: 1) greatest diameter of the CVC not overlapping the heart or diaphragm (that was at the $8^{\text {th }}$ intercostal space); 2) diameter of the descending aorta at the same intercostal space as the CVC measurement; 3) length of the thoracic vertebra over the tracheal bifurcation; and 4) diameter of the right fourth thoracic rib just ventral to the spine. Vessel diameters were measured perpendicular to the long axis of the vessel and thoracic vertebra length was measured at midbody (Fig. 1 \& 2 ). The measurements were made digitally with software. All obtained data were expressed in $\mathrm{cm}$. From these measurements, the ratios of $\mathrm{CVC} / \mathrm{Ao}, \mathrm{CVC} / \mathrm{R} 4, \mathrm{CVC} / \mathrm{VL}$, $\mathrm{CVC} / \mathrm{VL}$ of $\mathrm{RHF}$ cats to CVC/VL of healthy cats, and also ratio of $\mathrm{Ao} / \mathrm{VL}$ in both healthy and RHF cats were calculated. These ratios were selected based on some previous studies (Lehmkuhl et al.,

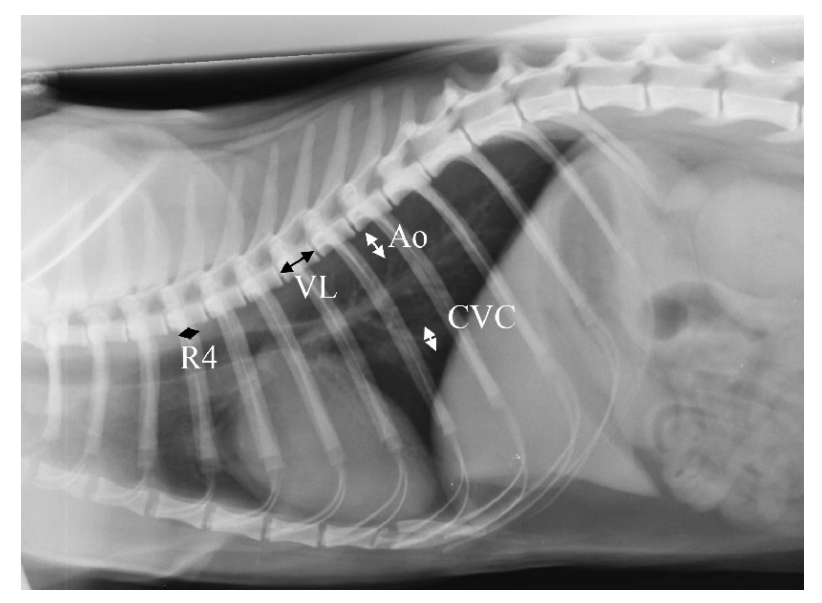

Fig. 1. Left lateral radiograph from a 5.5-year-old male DSH cat with right-sided congestive heart failure. Caudal vena cava (CVC), aorta (Ao), thoracic vertebral length (VL) and fourth rib width (R4). 


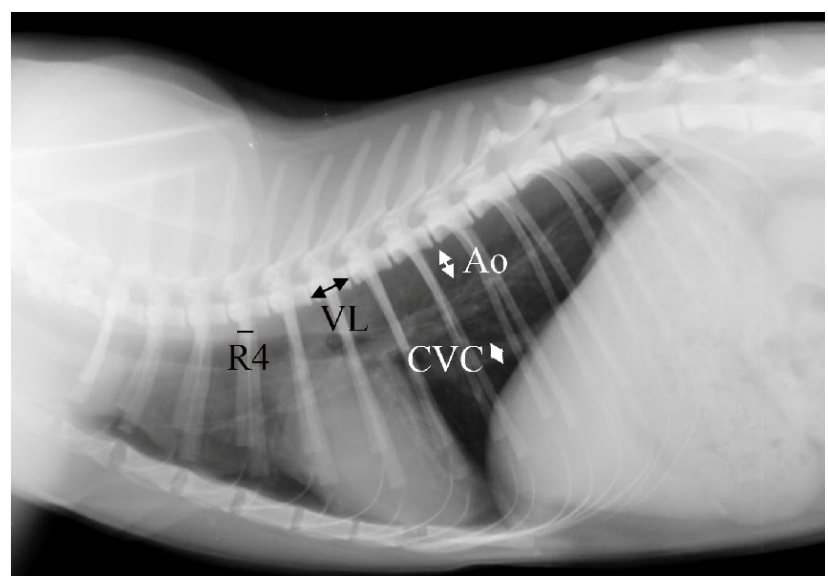

Fig. 2. Left lateral radiograph from a 6-year-old male DSH cat without heart failure. Caudal vena cava (CVC), aorta (Ao), thoracic vertebral length (VL) and fourth rib width (R4).

Table 1. Ratios of the caudal vena cava (CVC) to the aorta (Ao), vertebral length (VL) and fourth rib (R4) and ratio of aorta to vertebral length (mean \pm SE; $n=20)$

\begin{tabular}{lccc}
\hline & Healthy DSH cat & Right heart failure DSH cat & P value \\
\hline CVC/Ao & $0.77 \pm 0.02$ & $1.86 \pm 0.13$ & 0.001 \\
CVC/VL & $0.72 \pm 0.04$ & $1.21 \pm 0.05$ & 0.47 \\
CVC/R4 & $1.68 \pm 0.06$ & $3.12 \pm 0.29$ & 0.03 \\
Ao/VL & $0.95 \pm 0.03$ & $0.68 \pm 0.05$ & 0.96 \\
\hline
\end{tabular}

1997; Jilintal et al., 2006; Annette et al., 2005).

All obtained data were expressed as means \pm SE. Obtained data were analysed by Student's $t$-test followed by Tukey's test, using the software SPSS 16 (Statistical Package for the Social Sciences, version 16, SPSS; Chicago, USA). P-values less than 0.05 were considered statistically significant.

\section{RESULTS}

As shown in Table 1, the ratios of CVC to the aorta $(\mathrm{CVC} / \mathrm{Ao})$ in healthy and right heart failure DSH cats were $0.77 \pm 0.02$ and $1.86 \pm 0.13$ respectively. The ratio of $\mathrm{CVC}$ to diameter of $4^{\text {th }} \mathrm{rib}(\mathrm{CVC} / \mathrm{R} 4)$ in healthy group was $1.68 \pm 0.06$ while it was $3.12 \pm 0.29$ in RHF group. The ratio of $\mathrm{CVC}$ to the length of the thoracic vertebra (CVC/VL) was $0.72 \pm 0.04$ in the healthy cats while it was $1.21 \pm 0.05$ in the RHF ones. The independent t-test showed that the $\mathrm{CVC} / \mathrm{Ao}$ and $\mathrm{CVC} / \mathrm{R} 4$ ratios differed significantly between healthy and RHF groups $(\mathrm{P}<0.05)$. Although $\mathrm{CVC} / \mathrm{VL}$ ratio in RHF cats was higher than that in healthy cats, there was not a significant difference between them. On the other hand Ao/VL ratio in healthy group was higher than that in the RHF group $(\mathrm{P}>0.05)$. 
Radiological evaluation of caudal vena cava in domestic shorthair cats with regard to right heart...

\section{DISCUSSION}

Measurement of the CVC has been applied to assess right heart function (Aycock et al., 2014) and to monitor the effect of therapy in patients with congestive heart failure (Kaufman et al., 2005).

The physical diagnosis of right-sided congestive heart failure is based on the detection of elevated jugular venous pressure, abnormal jugular pulses, hepatosplenomegaly and ascites. A radiographic correlate to elevated right-sided cardiac pressures may be a dilated, tortuous or more radiopaque caudal vena cava (Lehmkuhl et al., 1997; Dew et al., 2005).

Size variability between dogs does not permit the comparison of absolute CVC size, but ratios of the CVC size to other anatomic structures may be useful to provide a quantitative assessment of CVC size. Quantitative analysis of the CVC in diseased dogs has only been reported twice. In both studies the diameter of the CVC was compared to the diameter of the fourth rib (R4) just below the spine in dogs with heartworm infestation (Losonsky et al., 1983; Thrall \& Calvert, 1983). A positive relation between the size of the CVC and right ventricle was found in dogs with heartworm infestation and the mean $\mathrm{CVC} / \mathrm{R} 4$ ratio was larger in dogs with congestive heart failure than in dogs without failure (Losonsky et al., 1983). Based on our results, both CVC/Ao and $\mathrm{CVC} / \mathrm{R} 4$ ratios were sensitive in DSH cats with right heart failure compare to the healthy ones.

In our study, DSH cats with right heart disease had larger caudal vena cava size than that in healthy DSH cats as determined by the ratios $\mathrm{CVC} / \mathrm{Ao}, \mathrm{CVC} / \mathrm{VL}$, and $\mathrm{CVC} / \mathrm{R} 4$.

The ratio of CVC/Ao may have been increased not only by an enlarged CVC but also by a small aorta (Jilintal et al.,
2006). In all radiographs, the aorta was subjectively small, and the Ao/VL ratio was smaller in DSH cats with right heart disease compared to the healthy DSH cats $(\mathrm{P}<0.05)$ (Table 1). One might speculate that a small aorta could occur due to decreased cardiac output associated with the underlying cardiac disease.

Despite a statistically significant difference between groups, overlap of control and abnormal data limit the clinical usefulness of these ratios to detect right heart disease in patients.

Likelihood ratios express the odds that a given ratio would be expected in a dog with a right-sided heart abnormality as opposed to one without (Sackett et al., 1991). For example Lehmkuhl et al. (1997) showed that a CVC/VL ratio of between 1.01 and 1.29 was twice as likely to come from a dog with a right-sided heart abnormality as from a healthy dog. In our study the $\mathrm{CVC} / \mathrm{VL}$ ratio in right heart failure DSH cats to $\mathrm{CVC} / \mathrm{VL}$ ratio in healthy DSH cats was 1.68. CVC/Ao and $\mathrm{CVC} / \mathrm{R} 4$ in RHF cats compare to the healthy cats were 2.42 and 1.86 respectively. These results showed that right heart failure cats had a caudal vena cava with more 1.5 times bigger than healthy cats.

Caudal vena cava size has been assessed via the $\mathrm{CVC} / \mathrm{R} 4$ ratio in some previous studies involving heartworm positive dog's heart failure. Based on these studies a larger $\mathrm{CVC} / \mathrm{R} 4$ ratio was reported in dogs with right-sided congestive heart failure from heartworm disease than that in heartworm infested dogs without congestive heart failure (Losonsky et al., 1983; Thrall \& Calvert, 1983). Our results about the $\mathrm{CVC} / \mathrm{R} 4$ ratio in the male DSH cats was in agreement with above studies in dogs. 
In a previous study by Lehmkuhl et al. (1997) the CVC was smaller than the length of the vertebra over the tracheal bifurcation in healthy dogs. Our result in the healthy male DSH cats was similar to it in dogs. In other words, the CVC/VL ratio in healthy dogs and cats is less than 1 compare to the RHF ones that is more than 1. CVC/VL ratios in our study in healthy and RHF cats were $0.72 \pm 0.04$ (less than 1) and 1.21 (more than 1) respectively.

Based on some studies, the Ao and VL values are related to growth; for example growing cows had significantly lower Ao and VL values than the adult cows (Jilintal et al., 2006). So it seems necessary to compare the same age animals as we did in this study.

The clinical utility of CVC dilation as an indicator of right heart abnormalities may be limited by individual variability and physiologic factors including phase of respiration, pleural pressure, phase of the cardiac cycle, and volume. We attempted to minimise the influence of respiration by only including peak inspiratory radiographs, but this factor cannot be excluded. Pleural pressure and phase of the cardiac cycle can obviously not be assessed in this study. Individual variability and the above mentioned physiologic factors may limit the predictive value of the ratios in this study. Additionally, measurement of the CVC and Ao is difficult to impossible in some patients. Poor radiographic quality, lung disease, pulmonary vasculature markings, cranial displacement of the diaphragm by ascites, or pleural fluid may obscure edge detection of the $\mathrm{CVC}$ and Ao.

In conclusion, the pulsation index of the $\mathrm{CVC}$ and the ratios of CVC to the diameter of the aorta and thoracic verte- bral length are useful parameters for diagnosis of cat with circulation dysfunction.

\section{REFERENCES}

Annette, L., A. Clarke, A. Rick \& B. James, 2005. Radiographic cardiac size in cats and dogs with heartworm disease compared with reference values using the vertebral heart scale method: 53 cases. Journal of Veterinary Cardiology, 7, 33-40.

Aycock, K. I., R. L. Campbell, K. B. Manning, S. P. Sastry, S. M. Shontz, F. C. Lynch \& B. A. Craven, 2014. A computational method for predicting inferior vena cava filter performance on a patient-specific basis. Journal of Biomechanical Engineering, 136, 081003.

Braun, U. \& T. Linggi, 1999. Ultrasonographic findings in three cows with chronic ragwort (Senecio alpinus) poisoning. The Veterinary Record, 144, 122-126.

Cina, A., G. Masselli, C. D. Stasi, L. Natale, A. R. Cotroneo, G. Cina \& L. Bonomo, 2006. Computed tomography imaging of vena cava filter complications: A pictorial review. Acta Radiologica, 47, 135-144.

Cuevas, C., M. Raske, W. H. Bush, T. Takayama, J. H. Maki, O. Kolokithas \& E. Meshberg, 2006. Imaging primary and secondary tumor thrombus of the inferior vena cava: multi-detector computed tomography and magnetic resonance imaging. Current Problems in Diagnostic Radiology, 35, 90-101.

Curvo-Semedo, L., L. Teixeira \& F. CaseiroAlves, 2005. Tuberculosis of the chest. European Journal of Radiology, 55, 158172.

Dew, J., K. Hansen, J. Hammon, T. McCoy, E. A. Levine \& P. Shen, 2005. Leiomyosarcoma of the inferior vena cava: Surgical management and clinical results. American Surgery, 71, 497-501.

Gidlewski, J. \& J. P. Petrie, 2005. Therapeutic pericardiocentesis in the dog and cat. 
Radiological evaluation of caudal vena cava in domestic shorthair cats with regard to right heart...

Clinical Techniques in Small Animal Practice, 20, 151-155.

Hollerbach, S., K. Schultze, M. Muscholl \& J. Schölmerich, 2001. Ultrasonography of the inferior vena cava (IVC) in the diagnosis and monitoring of therapy in patients with chronic congestive heart failure. Deutsche Medizinische Wochenschrift, 126, 129-133.

Jilintal, S. Hashiyama, Y. Gonda, H. Ishikawa, M. Sato \& K. Miyahara, 2006. Radiographic evaluation of caudal vena cava size as a useful parameter for the diagnosis of heart disease in dairy cattle. The Journal of Veterinary Medical Sciences, 68, 995-998.

Kaufman, L.B., B. M. Yeh, R. S. Breiman, B. N. Joe, A. Qayyum \& F. V. Coakley, 2005. Inferior vena cava filling defects on CT and MRI. American Journal of Roengenology, 185, 717-726.

Kieffer, E., M. Alaoui, J. C. Piette, P. Cacoub \& L. Chiche, 2006. Leiomyosarcoma of the inferior vena cava: Experience in 22 cases. Annals of Surgery, 244, 289-295.

Konig, L. H. \& H. G. Liebich, 2004. Veterinary Anatomy of Domestic Mammals. Schattauer, pp. 449-450.

Lehmkuhl, L. B., J. D. Bonagura, D. S. Biller \& W. M. Hartma, 1997. Radiographic evaluation of caudal vena cava size in dogs. Radiology and Ultrasound, 38, 94100 .

Losonsky, J.M., D. E. Thrall \& R. E. Lewis, 1983. Thoracic radiographic abnormalities in 200 dogs with spontaneous heartworm infestation. Veterinary Radiology and $\mathrm{Ul}$ trasound, 24, 120-123.

Lyon, M., M. Blaivas \& L. Brannam, 2005. Sonographic measurement of the inferior vena cava as a marker of blood loss. American Journal of Emergency Medicine, 23, 45-50.

Natori, H., S. Tamaki \& S. Kira, 1979. Ultrasonographic evaluation of ventilatory effect on inferior vena caval configuration. The American Review of Respiratory Disease, 120, 421-427.
Sackett, D. L., R. B. Haynes, G. H. Guyatt \& P. Tugwell, 1991. Clinical Epidemiology, $2^{\text {nd }}$ edn, Little, Brown and Company, Boston.

Sheth, S. \& E. K. Fishman, 2007. Imaging of the Inferior vena cava with MDCT. American Journal of Radiology, 189, 12431251.

Thrall, D. E. \& C. A. Calvert, 1983. Radiographic evaluation of canine heart-worm disease coexisting with right heart failure. Veterinary Radiology, 24, 124-126.

Wachsberg, R. H., C. D. Levine, P. D. Maldjian, \& M. Z. Simmons, 1998. Dilatation of the inferior vena cava in patients with cirrhotic portal hypertension: Causes and imaging findings. Clinical Imaging, 22, $48-53$.

Yeh, B. M., P. Kurzman, E. Foster, A. Qayyum, B. Joe \& F. Coakley, 2004. Clinical relevance of retrograde inferior vena cava or hepatic vein opacification during contrast-enhanced CT. American Journal of Roentgenology, 183, 1227-1232.

Paper received 30.04.2017; accepted for publication 05.06.2017

\section{Correspondence:}

Dariush Vosugh

Department of Clinical Sciences,

School of Veterinary Medicine,

Shahid Bahonar University of Kerman,

P.O Box: 7616914111 Kerman, Iran

tel: +983431322921

fax: +983433257250

e-mail: dvosugh@yahoo.com 